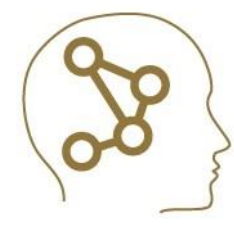

\title{
Modeling of Telecommunication Revenue as a Percentage of Gross Domestic Product's for Countries with Fractional Calculus
}

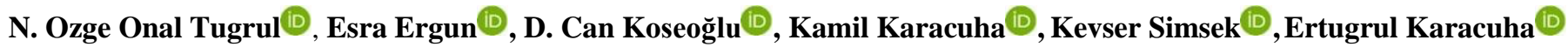 \\ Istanbul Technical University, Informatics Institute, Istanbul, Turkey. (e-mail: \{onal16, ergunesr, koseoglu20, karacuha17, simsek18, karacuhae\}@itu.edu.tr.
}

\section{ARTICLE INFO}

Received: March.,03.202

Revised: April,21.2021

Accepted: May.,17.2021

\section{Keywords:}

Fractional Calculus

GDP

MAPE

Modeling

Telecommunication Revenue

Corresponding author: Nisa Ozge

Onal Tugrul

$\triangle$ onal16@itu.edu.tr

湎 +90212 2857391

ISSN: $2548-0650$

DOI: https://doi.org/10.52876/jcs.911144

\begin{abstract}
This study explores the modeling of the share of telecommunication revenues in gross domestic product from the year 2000 to 2018 for 5 countries including France, Germany, Italy, Turkey, the UK, and the OECD average. First, a new mathematical model based on Fractional Calculus and Least Square Method is proposed. Later, the telecommunication revenues in GDP dataset is modeled. Further, we compare the new Fractional approach to the classical Polynomial approach in three different settings. The results show that employing Fractional Calculus yields better modeling performance when compared to the classical Polynomial Approach in terms of Mean Absolute Percentage Error (MAPE). The Fractional approach outperforms the Polynomial approach by $0.1329 \%$ MAPE on average. The largest MAPE is found for Turkey while the smallest MAPE is obtained for Italy in all settings.
\end{abstract}

\section{INTRODUCTION}

$\mathrm{T}$ ELECOMMUNICATIONS can be defined as the exchange of any signals such as written messages, images, icons, sounds, or information by utilizing various media such as wire, radio, optical, or other electromagnetic systems [1,2]. Early days of communication consist of visual and audial signals such as horns, drums, signal flakes, smoke signals. In $20^{\text {th }}$ and $21^{\text {th }}$ centuries, long-distance communication technologies evolved with the help of inventions such as the telegraph, the telephone, the radio, network, antenna systems, optical fiber, and communication satellites. In parallel, the demand for data exchange increased too. Therefore, understanding the changes of this demand and communication need in specific intervals are important and crucial for scientists, companies, and states for modeling and analyzing the pattern of progress.

In the last several decades, telecommunication need is increased drastically with the help of advancing technology. Communication systems used previously cannot support the state of the art technologies. Increasing data sizes and the need for reaching more than one user at a time leads to make progress. This progress has its advantages and disadvantages. One advantage is, communication quality and comfort have increased and the desired data can be accessed in a short time. The disadvantage is, legacy systems needs to be replaced by new technological tools which increases the cost for investors. These innovations or technology replacements affect firms and therefore the income of the countries. The expenses and revenues of the systems that have changed over the years constitute an important share of the countries' economy.

Previously, the "Marginal Revolution" and "Keynesian revolution" offered fundamental economic methods. Regarding these, the concepts of "marginal value", "economic multiplier", "economic accelerator", "elasticity" were studied [3-5]. These revolutions prompted the researchers, companies, and institutes to employ mathematical tools such as derivatives and integrals in modeling each specific case for their problem. Then, the economic models were understood and tested very easily with these equations including the differential, integral, or difference equations [3]. 
Integer-order derivatives and integrals are well-known and studied for centuries by researchers [3-8]. Fractional Calculus is the theory of non-integer or complex-valued derivatives and integrals [9-20]. The applications of such mathematical tools in practical and engineering problems are relatively new [311]. The fractional approach has the flexibility, hereditary, and dynamicity and therefore it can be applied to areas such as mathematical economics, management, and finance $[3,7$, 12]. The main goals, notions, effects, and objectives of mathematical economics can be generalized, widen, and improved by including such new approaches [3].

In this study, we propose a new mathematical model based on Fractional Calculus and model the telecommunication revenue as a percentage of GDP for 5 countries including France, Germany, Italy, Turkey, the UK, and the OECD average. We name this new approach as Fractional Model-2. Later, we assess the performance of the newly proposed approach with the help of conventional Polynomial model and compare these two models.

The structure of this study is as follows. Section 2 provides the foundations of the employed fractional model. Then, in Section 3, Dataset and Performance Metrics are presented. Later, Section 4 reports the experimental results and lastly, the conclusion is given in Section 5.

\section{MATHEMATICAL MODEL}

The main motivation is to model the given discrete dataset and obtained a continuous curve representing the dataset with the minimum error. To achieve this goal, the Taylor expansion is employed at the first stage of the mathematical manipulations [18-21].

An arbitrarily chosen, continuous and analytical function $g(x)$ can be expanded as

An arbitrarily chosen, continuous and analytical function $g(x)$ can be expanded as

$$
g(x)=\sum_{n=0}^{\infty} \tilde{a}_{n} x^{n+\alpha}
$$

Then, the first derivative of the function with respect to $\mathrm{x}$ becomes $g^{\prime}(x)=\sum_{n=0}^{\infty} \tilde{a}_{n}(n+\alpha) x^{n+\alpha-1}$. From (1), we would like to mimic the same approach for the function $f(x)$ which stands for the income of the telecommunication sector in years. Note that, in this case, $x$ corresponds to years. To have a better modeling approach utilizing the non-locality and heredity properties of fractional calculus, the fractional derivative of $f(x)$ is expressed as Equation (2).

$$
\frac{d^{\alpha} f(x)}{d x^{\alpha}}=\sum_{n=0}^{\infty} a_{n}(n+\alpha) x^{n+\alpha-1}
$$

Here, $\alpha$ is the fractional-order and ranges from [0,1] [21]. The main motive is to find $f(0), a_{n}$, and $\alpha$ representing $f(x)$ with minimum error. Before, going into details, it is better to define the fractional derivative. Caputo's definition of the fractional derivative is provided below [14, 18, 22].

$$
\mathfrak{D}_{x}^{\alpha} f(x)=\frac{d^{\alpha} f(x)}{d x^{\alpha}}=\frac{1}{\Gamma(1-\alpha)} \int_{-\infty}^{x} \frac{f^{\prime}(t)}{(x-t)^{\alpha}} d t
$$

where fractional derivative $\mathfrak{D}_{x}^{\alpha}$ states that the derivative is taken with respect to $x$ in the order of $\alpha(\alpha \in[0,1])$, and $f^{\prime}$ stands for the first derivative.

Note that, $\Gamma(1-\alpha)$ is called Gamma function and given as Equation (4)

$$
\Gamma(1-\alpha)=\int_{0}^{\infty} t^{-\alpha} e^{-t} d t
$$

By generalizing the derivative operator, more flexible and fast converging modeling becomes possible.

To solve the fractional-order differential equation given in Equation (2), the Laplace Transform is taken and the differential equation is converted into an algebraic equation. In Equation (5) and Equation (6), two properties of Laplace Transform $(\mathcal{L})$ are listed $[6,19,22]$.

$$
\begin{gathered}
x^{\alpha} \stackrel{\mathcal{L}}{\rightarrow} \frac{\Gamma(\alpha+1)}{s^{\alpha+1}} \\
\mathcal{L} \frac{d^{\alpha} f}{d x^{\alpha}}=\stackrel{\mathcal{L}}{\rightarrow} s^{\alpha} F(s)-s^{\alpha-1} f(0)
\end{gathered}
$$

Note that, $F(s)$ is the Laplace Transform of $f(x)$. The properties are employed in Equation (2) and the following procedure is tracked.

$$
\begin{gathered}
\mathcal{L} \frac{d^{\alpha} y}{d x^{\alpha}}=\mathcal{L} \sum_{n=0}^{\infty} a_{n}(n+\alpha) x^{n+\alpha-1} \\
s^{\alpha} F(s)-s^{\alpha-1} f(0) \\
=\sum_{n=0}^{\infty} a_{n}(n+\alpha) \frac{\Gamma(n+\alpha)}{s^{n+\alpha}} \Gamma(n+\alpha+1) \\
F(s)=s^{-1} f(0)+\sum_{n=0}^{\infty} a_{n} \frac{\Gamma(n+\alpha+1)}{s^{n+2 \alpha}}
\end{gathered}
$$

After obtaining the algebraic equation for $F(s)$ as given in Equation (7), the inverse Laplace Transform $\left(\mathcal{L}^{-1}\right)$ is employed to obtain $f(x)$ which is provided in Equation (8).

$$
f(x)=f(0)+\sum_{n=0}^{\infty} a_{n} \frac{\Gamma(n+\alpha+1)}{\Gamma(n+2 \alpha)} x^{n+2 \alpha-1}
$$

For the numerical calculation, the infinite sum is truncated to $N$ and approximate value of $f(x)$ is given in (9).

$$
f(x) \cong f(0)+\sum_{n=0}^{N} a_{n} \frac{\Gamma(n+\alpha+1)}{\Gamma(n+2 \alpha)} x^{n+2 \alpha-1}
$$

At this point, theoretically, $f(x)$ function is achieved. To obtain the unknowns $f(0), a_{n}$, and $\alpha$, the discrete dataset is employed and then, by error minimization, continuous $f(x)$ function representing that specific dataset would be acquired. The dataset consists of Telecommunication GDP income per year. Here, telecommunication GDP income was defined as $P_{i}$ and $x_{i}$ represents the telecommunication income in years as expressed below.

$$
\begin{aligned}
& P_{i}=\left[\begin{array}{llll}
p_{0} & p_{1} & \ldots & p_{K-1}
\end{array}\right] \\
& x_{i}=\left[\begin{array}{llll}
x_{0} & x_{1} & \ldots & x_{K-1}
\end{array}\right]
\end{aligned}
$$

Note that $K$ values exist. At this point, function $f\left(x_{i}\right)$ will be the expected value for $x_{i}^{t h}$ year. According to the least square 
method $\epsilon_{i}$, which is defined as the error between $p_{i}$ and $f\left(x_{i}\right)$ values, is shown as follows.

$$
\left(\epsilon_{i}\right)^{2}=\left(p_{i}-f\left(x_{i}\right)\right)^{2}
$$

The total square of the error is defined as Equation (11) and according to the least-squares method, the sum of error squares $\epsilon_{T}^{2}$ is tried to be minimized [18-20].

$$
\epsilon_{T}^{2}=\epsilon_{0}^{2}+\epsilon_{1}^{2}+\epsilon_{2}^{2}+\cdots \epsilon_{K-1}^{2}=\sum_{i=0}^{K-1} \epsilon_{i}^{2}
$$

For the sake of simplicity, the square of error for each point in the dataset can be obtained as follows:

$$
\begin{aligned}
& \left(\epsilon_{0}\right)^{2}=\left[p_{0}-\left\{f(0)+\sum_{n=0}^{N} a_{n} \frac{\Gamma(n+\alpha+1)}{\Gamma(n+2 \alpha)} x_{0}{ }^{n+2 \alpha-1}\right\}\right]^{2} \\
& \left(\epsilon_{1}\right)^{2}=\left[p_{1}-\left\{f(0)+\sum_{n=0}^{N} a_{n} \frac{\Gamma(n+\alpha+1)}{\Gamma(n+2 \alpha)} x_{1}{ }^{n+2 \alpha-1}\right\}\right]^{2} \\
& \epsilon_{i}^{2}=\sum_{i=0}^{K-1}\left[p_{i}-\left\{f(0)+\sum_{n=0}^{N} a_{n} \frac{\Gamma(n+\alpha+1)}{\Gamma(n+2 \alpha)} x_{i}{ }^{n+2 \alpha-1}\right\}\right]^{2} \\
& \left(\epsilon_{K-1}\right)^{2}=\left[p_{K-1}-\left\{f(0)+\sum_{n=0}^{N} a_{n} \frac{\Gamma(n+\alpha+1)}{\Gamma(n+2 \alpha)} x_{K-1}{ }^{n+2 \alpha-1}\right\}\right]^{2}
\end{aligned}
$$

For minimizing the total error given in Equation (11), the Least Squares Method is employed as given in $(13)[18,21]$ :

$$
\frac{\partial \epsilon_{T}^{2}}{\partial f(0)}=0, \quad \frac{\partial \epsilon_{T}^{2}}{\partial a_{0}}=0, \quad \frac{\partial \epsilon_{T}^{2}}{\partial a_{1}}=0, \quad \frac{\partial \epsilon_{T}^{2}}{\partial a_{2}}=0, \quad \frac{\partial \epsilon_{T}^{2}}{\partial a_{N}}=0
$$

Implementing Equation (13) leads to having $N+2$ equations and also Equation (9) has the same number of unknowns. Therefore, this problem can be solved. The Least Squares method leads to having a System of Linear algebraic equations (SLAE). Several specific derivative operations in Equation (13) are given below for the readers.

First example:

$$
\begin{aligned}
\frac{\partial \epsilon_{T}^{2}}{\partial f(0)} & =-2\left[p_{0}-\left\{f(0)+\sum_{n=0}^{N} a_{n} \frac{\Gamma(n+\alpha+1)}{\Gamma(n+2 \alpha)} x_{0}^{n+2 \alpha-1}\right\}\right] \\
& -2\left[p_{1}-\left\{f(0)+\sum_{n=0}^{N} a_{n} \frac{\Gamma(n+\alpha+1)}{\Gamma(n+2 \alpha)} x_{1}^{n+2 \alpha-1}\right\}\right] \\
-2 & {\left[p_{2}-\left\{f(0)+\sum_{n=0}^{N} a_{n} \frac{\Gamma(n+\alpha+1)}{\Gamma(n+2 \alpha)} x_{2}^{n+2 \alpha-1}\right\}\right] } \\
& -2\left[p_{K-1}-\left\{f(0)+\sum_{n=0}^{N} a_{n} \frac{\Gamma(n+\alpha+1)}{\Gamma(n+2 \alpha)} x_{K-1}^{n+2 \alpha-1}\right\}\right]=0
\end{aligned}
$$

Then, the procedure above can be written in the compact form as Equation (14):

$$
\begin{gathered}
\frac{\partial \epsilon_{T}^{2}}{\partial f(0)}=\sum_{i=0}^{K} p_{i}-\left[(K+1) f(0)+a_{0} \frac{\Gamma(\alpha+1)}{\Gamma(2 \alpha)} \sum_{i=0}^{K} x_{i}^{2 \alpha-1}\right. \\
+a_{1} \frac{\Gamma(\alpha+2)}{\Gamma(2 \alpha+1)} \sum_{i=0}^{K} x_{i}^{2 \alpha}+\cdots \\
\left.+a_{N} \frac{\Gamma(N+\alpha+1)}{\Gamma(N+2 \alpha)} \sum_{i=0}^{K-1} x_{N}^{N+2 \alpha-1}\right] \\
=0
\end{gathered}
$$

Second Example:

$$
\begin{aligned}
\frac{\partial \epsilon_{T}^{2}}{\partial a_{N}}=-2\left[p_{0}-\left\{f(0)+\sum_{n=0}^{N} a_{n} \frac{\Gamma(n+\alpha+1)}{\Gamma(n+2 \alpha)} x_{0}^{n+2 \alpha-1}\right\}\right] x_{0}^{N+2 \alpha-1} \\
-2\left[p_{1}\right. \\
\left.-\left\{f(0)+\sum_{n=0}^{N} a_{n} \frac{\Gamma(n+\alpha+1)}{\Gamma(n+2 \alpha)} x_{1}^{n+2 \alpha-1}\right\}\right] x_{1}^{N+2 \alpha-1} \\
-2\left[p_{2}\right. \\
\left.-\left\{f(0)+\sum_{n=0}^{N} a_{n} \frac{\Gamma(n+\alpha+1)}{\Gamma(n+2 \alpha)} x_{2}^{n+2 \alpha-1}\right\}\right] x_{2}^{N+2 \alpha-1} \\
-2\left[p_{K-1}-\left\{f(0)+\sum_{n=0}^{N} a_{n} \frac{\Gamma(n+\alpha+1)}{\Gamma(n+2 \alpha)} x_{K-1}^{n+2 \alpha-1}\right\}\right] x_{K-1}^{N+2 \alpha-1} \\
=0
\end{aligned}
$$

Then, the procedure above can be summarized in the compact form as Equation (15):

$$
\begin{aligned}
\frac{\partial \epsilon_{T}^{2}}{\partial a_{N}}=\left[\sum_{i=0}^{K} p_{i} x_{i}^{N+2 \alpha-1}\right] & \\
& -\left[f(0) \sum_{i=0}^{K} x_{i}^{N+2 \alpha-1}+a_{0} \frac{\Gamma(\alpha+1)}{\Gamma(2 \alpha)} \sum_{i=0}^{K} x_{i}^{4 \alpha+N-2}\right. \\
& +a_{1} \frac{\Gamma(\alpha+2)}{\Gamma(2 \alpha+1)} \sum_{i=0}^{K} x_{i}^{4 \alpha+N-1}+\cdots \\
& \left.+a_{N} \frac{\Gamma(N+\alpha+1)}{\Gamma(N+2 \alpha)} \sum_{i=0}^{K} x_{i}^{4 \alpha+2 N-2}\right]=0
\end{aligned}
$$

The procedure is repeated for all cases in Equation (13). Then, the following SLAE is achieved.

$$
[A]_{N+2 \times N+2}[\Omega]_{N+2 \times 1}=[B]_{N+2 \times 1}
$$

Here,

$$
\begin{aligned}
& A=\left[\begin{array}{ccccc}
K+1 & \sum_{i=1}^{k} c_{0}\left(x_{i}\right) & \sum_{i=1}^{k} c_{1}\left(x_{i}\right) & \cdots & \sum_{i=1}^{k} c_{N}\left(x_{i}\right) \\
\sum c_{0}\left(x_{i}\right) & \sum c_{0}\left(x_{i}\right) c_{0}\left(x_{i}\right) & \sum c_{0}\left(x_{i}\right) c_{1}\left(x_{i}\right) & \cdots & \sum^{2} c_{0}\left(x_{i}\right) c_{N}\left(x_{i}\right) \\
\sum c_{1}\left(x_{i}\right) & \sum c_{1}\left(x_{i}\right) c_{0}\left(x_{i}\right) & \sum c_{1}\left(x_{i}\right) c_{1}\left(x_{i}\right) & \cdots & \sum c_{1}\left(x_{i}\right) c_{N}\left(x_{i}\right) \\
\vdots & \vdots & \vdots & \vdots \\
\sum c_{m}\left(x_{i}\right) & \sum c_{m}\left(x_{i}\right) c_{0}\left(x_{i}\right) & \sum c_{m}\left(x_{i}\right) c_{1}\left(x_{i}\right) & \cdots & \sum_{i=1}^{k} c_{m}\left(x_{i}\right) c_{N}\left(x_{i}\right)
\end{array}\right] \\
& {[\Omega]=\left[\begin{array}{lllll}
f(0) & a_{0} & a_{1} & \ldots & a_{N}
\end{array}\right]^{T}}
\end{aligned}
$$

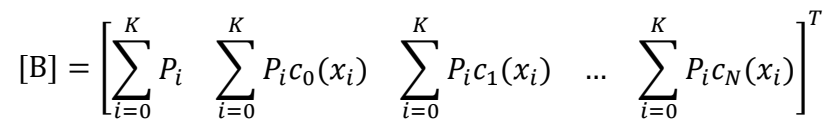

where, 


$$
c_{m}(x, \alpha)=\frac{\Gamma(m+\alpha+1)}{\Gamma(m+2 \alpha)} x^{m+2 \alpha-1}
$$

Here, $m=1,2, \ldots N$

The vector $\Omega$ consist of unknowns $\left(f(0), a_{n}\right)$. By inversion of $[A], \Omega$ vector can be obtained. Then, Equation (9) allows one to obtain $f(x)$ which represents the discrete dataset with minimum error. The optimum value of $\alpha$ is found by implementing a grid search. Note that, when the fractional order $\alpha$ is equal to one, the fractional approach is equal to the polynomial method.

\section{DATASET AND PERFORMANCE METRICS}

In this study, we model the telecommunication revenues as a percentage of GDP for countries and compare them from the year 2000 to 2018. The dataset of the telecommunication revenues for each country is extracted from OECD [24]. The dataset is reported in Figure 1 and Table A.1 of the Appendix for five countries (France, Germany, Italy, Turkey, and UK) and the average of the OECD members.

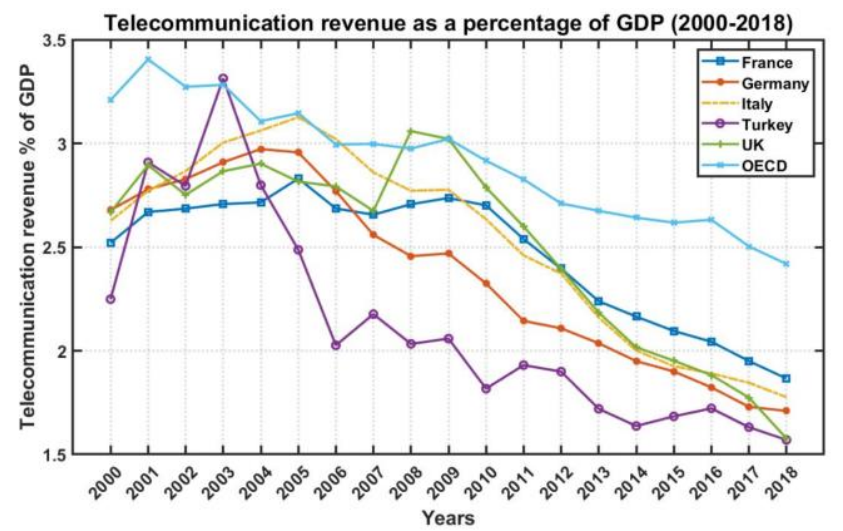

Fig.1. Telecommunication revenues as a percentage of GDP of the countries.

It is important to know that these selected countries are comparable and similar regarding the population, the number of subscribers, technological infrastructure. However, as expected, there are also differences among the selected countries such as the percentage of young people or adults over the total population which can affect the revenue of the telecommunication sector and total economical size of the country. Nevertheless, a key point in the present study is how the telecommunication share affects the countries economy. All the results reported in tables are in terms of Mean Absolute Percentage Error (MAPE). The MAPE is calculated as in (19).

$$
M A P E=\frac{1}{n} \sum_{i=1}^{n}\left|\frac{P(i)-f\left(x_{i}\right)}{P(i)}\right| \times 100
$$

The average error in all percentiles is calculated by the formula in (20)

$$
A M A P E=\frac{\sum M A P E}{M}
$$

\section{EXPERIMENTAL RESULTS}

This section will provide modeling results of Fractional and Polynomial methods with three different modeling settings. Each set has a different exponent value. Table 1 illustrates the modeling results of three $N$ 's for both fractional and polynomial models. When $N=5$, the performance of the fractional approach outperforms the polynomial approach by $0.3152 \%$ MAPE where the first model yields $2.2909 \%$ and the latter yields $2.6061 \%$ AMAPE.

When the exponent is 8 , the fractional approach produces \%1.7202 AMAPE where the polynomial approach yields $1.7861 \%$ AMAPE. Lastly, when the exponent is equal to 10 , the fractional approach results in $1.7031 \%$ MAPE while the polynomial method results in $1.7208 \%$ AMAPE.

TABLE I

\begin{tabular}{|c|c|c|c|c|c|c|c|c|}
\hline $\begin{array}{l}\frac{0}{3} \\
\frac{\pi}{2} \\
z\end{array}$ & $\begin{array}{l}\frac{\infty}{0} \\
\frac{0}{0} \\
\sum\end{array}$ & 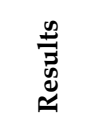 & 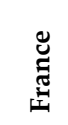 & $\underset{\Xi}{\stackrel{\Xi}{\Xi}}$ & $\stackrel{\text { त }}{\stackrel{ \pm}{=}}$ & $\underset{\vec{\theta}}{\vec{y}}$ & 光 & 己ి \\
\hline \multirow{3}{*}{$\begin{array}{l}\text { LI } \\
\text { Z }\end{array}$} & Fract. & MAPE & 1.69 & 1.59 & 1.33 & 4.12 & 3.59 & 1.40 \\
\hline & Model & $\alpha$ & 0.97 & 1 & 0.50 & 0.50 & 0.95 & 0.50 \\
\hline & $\begin{array}{l}\text { Pol. } \\
\text { Model }\end{array}$ & MAPE & 1.70 & 1.59 & 1.44 & 5.80 & 3.61 & 1.50 \\
\hline \multirow{3}{*}{$\begin{array}{l}\infty \\
\text { II }\end{array}$} & Fract. & MAPE & 1.16 & 1.23 & 1.05 & 3.71 & 2.13 & 1.01 \\
\hline & Model & $\alpha$ & 0.50 & 0.50 & 0.92 & 0.50 & 0.50 & 1 \\
\hline & $\begin{array}{l}\text { Pol. } \\
\text { Model }\end{array}$ & MAPE & 1.25 & 1.28 & 1.07 & 3.88 & 2.22 & 1.02 \\
\hline \multirow{3}{*}{$\begin{array}{l}\text { 육 } \\
\text { Z }\end{array}$} & Fract. & MAPE & 0.76 & 0.89 & 0.59 & 3.24 & 1.59 & 0.67 \\
\hline & Model & $\alpha$ & 0.50 & 0.82 & 1 & 0.96 & 0.50 & 0.50 \\
\hline & $\begin{array}{l}\text { Pol. } \\
\text { Model }\end{array}$ & MAPE & 0.82 & 0.93 & 0.60 & 3.25 & 1.94 & 0.80 \\
\hline
\end{tabular}

MODELING RESULTS OF THE TELECOMMUNICATION REVENUE AS A PERCENTAGE OF GDP (2000-2018).

For all three settings, the Fractional approach outperforms the polynomial approach. Also, for all exponent values, the largest MAPE is observed in Turkey and the smallest is observed for Italy. As expected, increasing the exponent value decreases the error rate.

The largest MAPE difference between the two models is observed when $N=5$. Note that, when the fractional order $\alpha$ is equal to one, the fractional approach is equal to the polynomial approach. For Germany where $N=5$, for OECD average where $N=8$, and lastly for Italy where $N=10$, the optimized fractional order is found as 1 . In these three cases, the MAPE results of the two models are equal as reported in Table 1.

Figures 2, 3, and 4 illustrate the actual and the modeled data curves for both Fractional Model and Polynomial Model. In most cases, Fractional and Polynomial modeled curves are similar to each other. The biggest difference is observed for Turkey in Figure 2. As seen from the plot, the fractional model fits the data better. This is consistent with the MAPE results reported in Table 1. It can be seen from Figure 1, the Polynomial and Fractional Models produce similar results. Numerically, Italy has the highest revenue USD in millions among the others.

From the figures, one can see that Italy has the highest telecommunication revenue percentage while Turkey has the lowest revenue percentage among others in 2000. Germany started with 2.6 percent telecommunication revenue and decreased to $1.7 \%$. Initially, Italy had $2.6 \%$ revenue in 2000 and decreased to around $1.77 \%$. France started with $2.5 \%$ revenue and ended up at $1.8 \%$. Turkey started with $2.24 \%$ revenue and decreased to $1.57 \%$. As seen from the figure, the telecommunication revenue \% of GDP decreased for all 
modeled countries and OECD average. The largest difference in percentage from 2000 to 2018 is observed for the UK. Also, Italy's trend is smoother compared to the others.

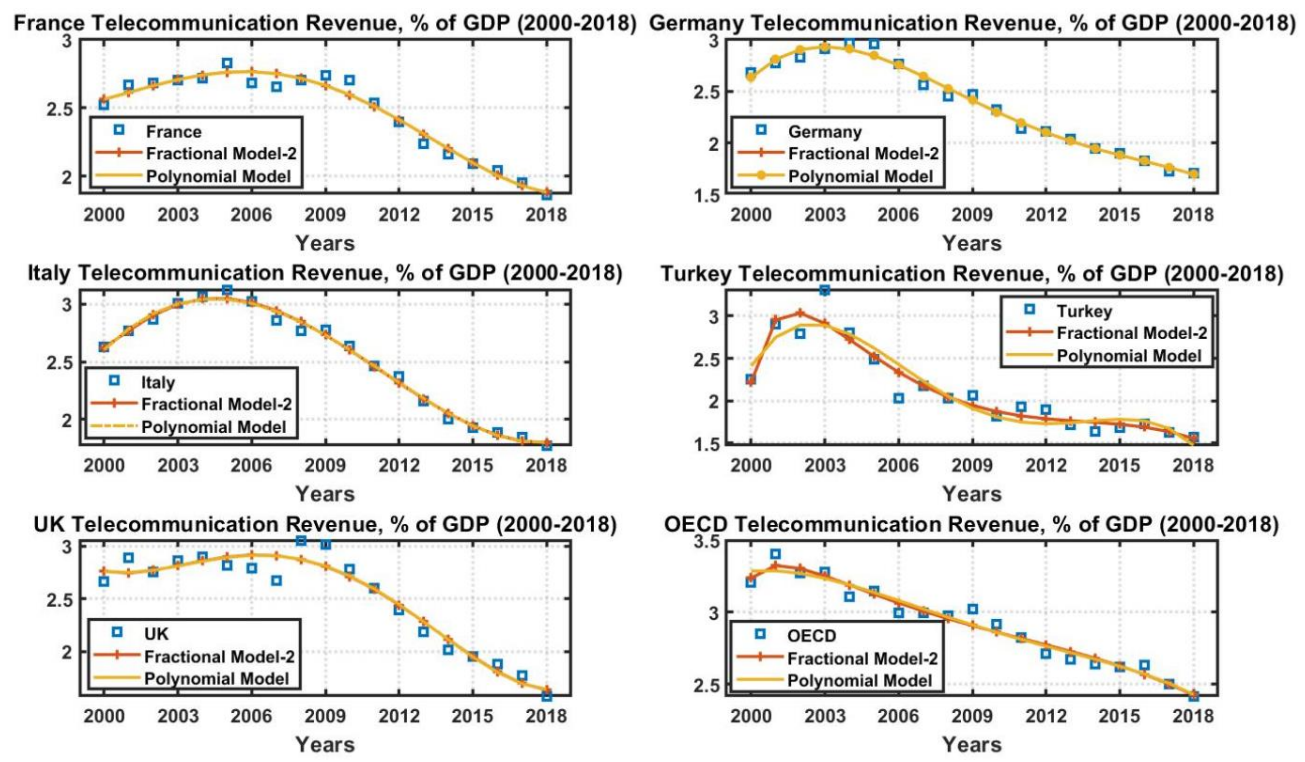

Fig. 2. Modeling of the countries using the Fractional model for $N=5$.

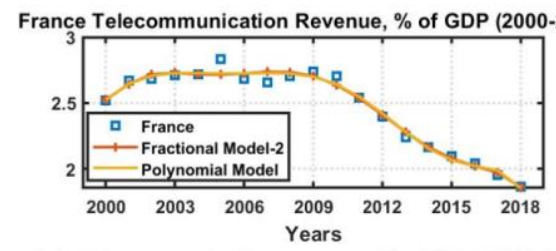

Italy Telecommunication Revenue, \% of GDP (2000-2018)

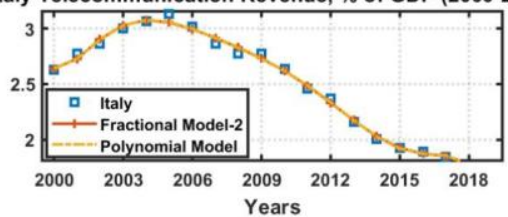

UK Telecommunication Revenue, \% of GDP (2000-2018)

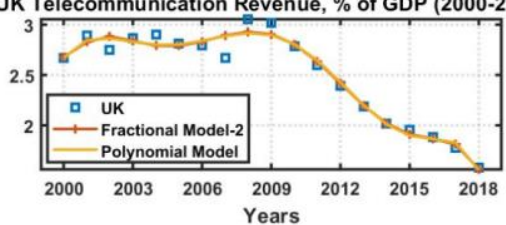

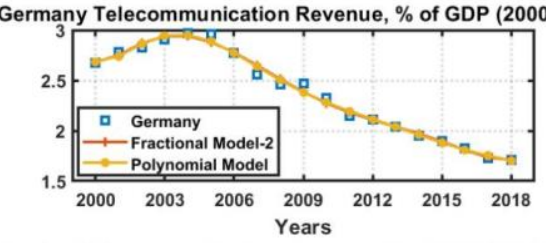

Turkey Telecommunication Revenue, \% of GDP (2000-2018)

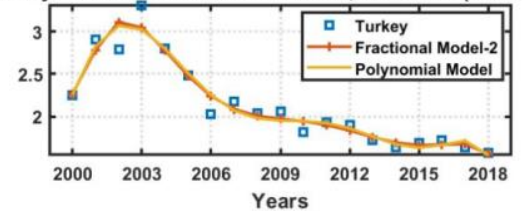

OECD Telecommunication Revenue, \% of GDP (2000-2018)

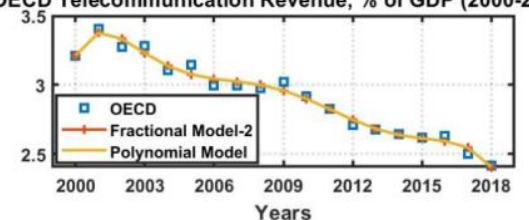

Fig. 3. Modeling of the countries using the Fractional model for $N=8$.

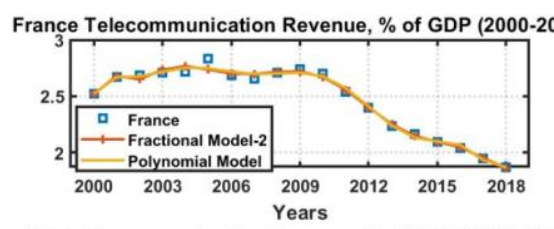

Italy Telecommunication Revenue, \% of GDP (2000-2018)

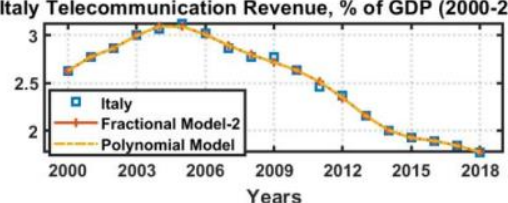

UK Telecommunication Revenue, \% of GDP (2000-2018)

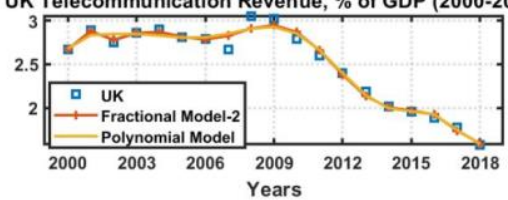

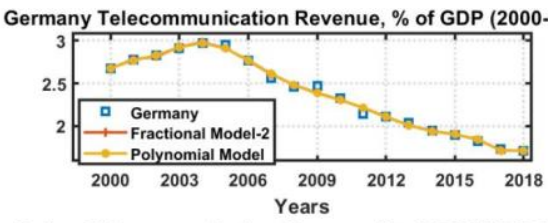

Turkey Telecommunication Revenue, \% of GDP (2000-2018)

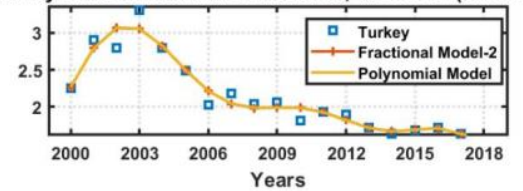

OECD Telecommunication Revenue, \% of GDP (2000-2018)

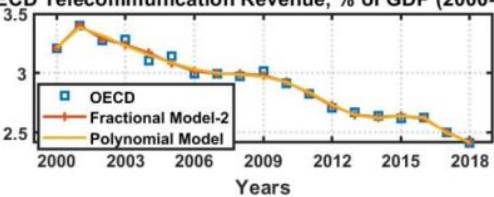

Fig. 4. Modeling of the countries using the Fractional model for $N=10$. 


\section{CONCLUSIONS}

In this study, we employed Fractional Calculus to model the telecommunications revenue as a percentage of the Gross Domestic Product of 5 countries (France, Germany, Italy, Turkey, and the UK) and OECD average. First, we proposed a new Fractional mathematical model that employs Least Square Methods named Fractional Model-2. Later, we compared the performances of the Fractional Model-2 and the Polynomial model. Results are reported for three experimental settings with three different exponent $N$ values $(5,8,10)$. As expected, increasing the exponent value decreased the error rate for both models. For all three settings, Fractional Approach resulted in better performance compared to the Polynomial Approach. The largest modeling error is obtained for Turkey while the smallest modeling error is observed in Italy. On average, the Fractional approach is superior to the Polynomial approach with $0.1329 \%$ MAPE.

\section{APPENDIX}

TABLE A.I

TELECOMMUNICATION REVENUE AS A PERCENTAGE OF GDP (2000-2018).

\begin{tabular}{|c|c|c|c|c|c|c|}
\hline 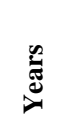 & 这 & हैं & 坣 & 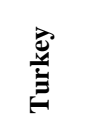 & 光 & 己ิ \\
\hline 2000 & 2.520 & 2.678 & 2.626 & 2.249 & 2.668 & 3.209 \\
\hline 2001 & 2.669 & 2.779 & 2.772 & 2.908 & 2.894 & 3.404 \\
\hline 2002 & 2.685 & 2.828 & 2.866 & 2.794 & 2.752 & 3.272 \\
\hline 2003 & 2.707 & 2.909 & 3.004 & 3.313 & 2.866 & 3.282 \\
\hline 2004 & 2.715 & 2.972 & 3.062 & 2.798 & 2.902 & 3.106 \\
\hline 2005 & 2.830 & 2.956 & 3.126 & 2.487 & 2.815 & 3.145 \\
\hline 2006 & 2.686 & 2.770 & 3.020 & 2.026 & 2.792 & 2.995 \\
\hline 2007 & 2.656 & 2.559 & 2.8597 & 2.1767 & 2.675 & 2.997 \\
\hline 2008 & 2.707 & 2.456 & 2.771 & 2.033 & 3.059 & 2.974 \\
\hline 2009 & 2.736 & 2.469 & 2.776 & 2.059 & 3.021 & 3.021 \\
\hline 2010 & 2.701 & 2.324 & 2.634 & 1.817 & 2.787 & 2.916 \\
\hline 2011 & 2.538 & 2.144 & 2.459 & 1.931 & 2.599 & 2.826 \\
\hline 2012 & 2.396 & 2.108 & 2.372 & 1.899 & 2.394 & 2.710 \\
\hline 2013 & 2.239 & 2.036 & 2.160 & 1.720 & 2.184 & 2.674 \\
\hline 2014 & 2.165 & 1.950 & 2.001 & 1.637 & 2.017 & 2.642 \\
\hline 2015 & 2.095 & 1.900 & 1.925 & 1.684 & 1.952 & 2.617 \\
\hline 2016 & 2.043 & 1.823 & 1.891 & 1.722 & 1.883 & 2.631 \\
\hline 2017 & 1.950 & 1.730 & 1.845 & 1.631 & 1.774 & 2.503 \\
\hline 2018 & 1.866 & 1.710 & 1.776 & 1.570 & 1.577 & 2.418 \\
\hline
\end{tabular}

\section{ACKNOWLEDGMENT}

This work is supported in part by Istanbul Technical University (ITU) Vodafone Future Lab under Project No. ITUVF20180901P11.

\section{REFERENCES}

[1] Regulations, R. International Telecommunication Union Std., November 2012. Online Available: http://www. itu. int/pub. RREGRR/en. (accessed 7 June 2020).

[2] ITU. Constitution and Convention of the International Telecommunication Union. Online Available: http://handle.itu.int/11.1004/020.2000/s.020, (accessed 9 June 2020).

[3] Tarasov, V. E. (2019). On history of mathematical economics: Application of fractional calculus. Mathematics, 7(6), 509.

[4] Schumpeter, J. A., \& Joseph, A. (1908). The Nature and Essence of Theoretical Economics.
[5] Hayes, M. (2008). The Economics of Keynes: A new guide to the General Theory. Edward Elgar Publishing.

[6] Samko, S. G., Kilbas, A. A., \& Marichev, O. I. (1993). Fractional Integrals and Derivatives-Theory and Applications Gordon and Breach. Linghorne, PA.

[7] Kiryakova, V. (1994). Generalized fractional calculus and applications longman (pitman res. notes in math. ser. 301).

[8] Podlubny, I. (1998). Fractional differential equations: an introduction to fractional derivatives, fractional differential equations, to methods of their solution and some of their applications. Elsevier.

[9] Kilbas, A. A., Srivastava, H. M., \& Trujillo, J. J. (2006). Theory and applications of fractional differential equations (Vol. 204). elsevier.

[10] Diethelm, K. (2010). The analysis of fractional differential equations: An application-oriented exposition using differential operators of Caputo type. Springer Science \& Business Media.

[11] Letnikov, A. V. (1868). On the historical development of the theory of differentiation with arbitrary index. Sbornik Mathematics (Matematicheskii Sbornik), 3(2), 85-112.

[12] Ross, B. (1975). A brief history and exposition of the fundamental theory of fractional calculus. In Fractional calculus and its applications (pp. 1-36). Springer, Berlin, Heidelberg.

[13] Ross, B. (1977). The development of fractional calculus 16951900. Historia Mathematica, 4(1), 75-89.

[14] Ross, B. (1977). Fractional calculus. Mathematics Magazine, 50(3), 115-122.

[15] Kiryakova, V. (2008). A brief story about the operators of the generalized fractional calculus. Fractional Calculus and Applied Analysis, 11(2), 203p-220p.

[16] Machado, J. T., Kiryakova, V., \& Mainardi, F. (2011). Recent history of fractional calculus. Communications in nonlinear science and numerical simulation, 16(3), 1140-1153.

[17] Machado, J. T., Galhano, A., \& Trujillo, J. (2013). Science metrics on fractional calculus development since 1966. Fractional Calculus and Applied Analysis, 16(2), 479-500.

[18] Önal, N. Ö., Karaçuha, K., Erdinç, G. H., Karaçuha, B. B., \& Karaçuha, E. (2019). A mathematical approach with fractional calculus for the modelling of children's physical development. Computational and mathematical methods in medicine, 2019.

[19] Karaçuha, E., Önal, N. Ö., Ergün, E., Tabatadze, V., Alkaș, H., Karaçuha, K., ... \& Nu, N. V. N. (2020). Modeling and Prediction of the Covid-19 Cases With Deep Assessment Methodology and Fractional Calculus. Ieee Access, 8, 164012-164034.

[20] Karaçuha, E., Tabatadze, V., Karacuha, K., Önal, N. Ö., \& Ergün, E. (2020). Deep assessment methodology using fractional calculus on mathematical modeling and prediction of gross domestic product per capita of countries. Mathematics, 8(4), 633.

[21] Herrmann, R. (2011). Fractional calculus: an introduction for physicists.

[22] OECD Key ICT Indicators. Telecommunication Revenue, Online Available: oecd.org/internet/broadband/oecdkeyictindicators.htm (accessed 25 Feb 2020).

[23] Worldbank World Development Indicators. GDP (current US\$). Online Available: https://databank.worldbank.org/source/world-developmentindicators (accessed 25 Feb 2020).

[24] De Myttenaere, A., Golden, B., Le Grand, B., \& Rossi, F. (2016). Mean absolute percentage error for regression models. Neurocomputing, 192, $38-48$.

\section{BIOGRAPHIES}

Nisa Ozge Onal Tugrul graduated from the School of Business at Istanbul University in 2016. She completed her M.Sc. degree Department of Applied Informatics from Istanbul Technical University in 2018. She is a research assistant and Ph.D. candidate at Istanbul Technical University in Turkey. Her research area is computational and applied mathematics.

Esra Ergun received the bachelor's and the master's degrees in Electronics and Communications Engineering and Computer Science departments from Istanbul Technical University, in 2017 and 2020 , respectively. She is currently pursuing a $\mathrm{Ph} . \mathrm{D}$. degree in Computer Science. She is also a Research Assistant with Istanbul Technical University. Her research interests include continual learning, representation learning, and domain adaptation for deep neural networks.

Deniz Can Koseoglu was born in Hatay, Turkey, in 1997. He received the B.Sc. degree from Electronics and Communications Engineering, Kocaeli University, Kocaeli, in 2019. He is currently a VOIP engineer 
in VTEL Communications and a Master's student at Istanbul Technical University.

Kamil Karacuha was born in Istanbul, Turkey, in 1993. He got his B.Sc. and double major degrees from Electrical \& Electronics and physics departments of the Middle East Technical University (Ankara) in 2017, 2018, respectively. He is working on Electromagnetic Theory, Scattering and Diffraction Problems in Electromagnetics, Antenna design, and mathematical modeling. Currently, he is a research assistant at Istanbul Technical University.

Kevser Simsek received a bachelor's degree in electronics and communications engineering from Sakarya University, in 2005. She received the M.Sc. degree from the Department of Electrical Engineering, New York University, USA, in 2010. Now, she is currently pursuing a Ph.D. degree with the Department of Applied Informatics, Informatics Institute, Istanbul Technical University. She is also working as senior data analyst at Turkish Airlines Inc. Her research interests include data analytics, machine learning, and domain adaptation for deep neural networks.

Ertugrul Karacuha was graduated from Electronics and Telecommunication Engineering department of Istanbul Technical University in 1986. He received his master's and doctorate degrees from Istanbul Technical University, Cukurova University, and Istanbul University in 1990, 1992, 1993, and 1996 respectively. From 19881989, He worked as a research \& development engineer in Teletaş, between 1989 and 1996 worked as a research assistant in Istanbul Technical University. From 1994 to 2001, Karaçuha was an assistant professor at Gebze Advanced Technology Institute. Between 2002 and 2013, he was the head of tariffs department and vice-president of Information and Communication Technologies Authority. He has been with Informatics Institute of Istanbul Technical University where he was appointed as a Professor and currently is the Dean of Informatics Institute. 